\title{
e-Migrinter
}

$12 \mid 2014$

Les chiffres de l'immigration : un regard critique sur les statistiques

\section{La construction des chiffres}

Marianne Blidon et Luc Legoux

\section{(2) OpenEdition}

Journals

Édition électronique

URL : https://journals.openedition.org/e-migrinter/345

DOI : 10.4000/e-migrinter.345

ISSN : 1961-9685

Éditeur

UMR 7301 - Migrinter

Édition imprimée

Date de publication : 15 octobre 2014

Pagination : $3-5$

ISSN : 1961-9685

\section{Référence électronique}

Marianne Blidon et Luc Legoux, "La construction des chiffres », e-Migrinter [En ligne], 12 | 2014, mis en ligne le , consulté le 20 mai 2021. URL : http://journals.openedition.org/e-migrinter/345 ; DOI : https:// doi.org/10.4000/e-migrinter.345 


\section{La construction des chiffres}

\section{Marianne Blidon \& Luc Legoux}

es statistiques migratoires, comme beaucoup de statistiques sociales, sont à la fois un outil de connaissance scientifique et de communication politique. En France, et plus généralement en Europe, où les migrations occupent depuis longtemps une place importante dans le débat public, le poids de la communication politique tend à être prépondérant et les chiffres sont souvent utilisés de manière a-critique. Dans ce contexte, l'étude détaillée de la construction des chiffres devient un élément nécessaire à l'analyse des discours sur l'immigration et plus généralement, à la compréhension des mouvements migratoires.
En effet, certains choix, dits "techniques », déterminent très fortement les utilisations possibles des résultats: les méthodologies d'enquête, la définition des catégories utilisées, les modes de calcul des estimations et la prise en compte des sources administratives, tous ces éléments permettent de mettre en évidence - ou à l'inverse, d'occulter - certaines caractéristiques des migrations. Ce dernier aspect est fondamental, l'absence de chiffre étant aussi un élément de communication politique très performant.

Ce numéro d'e-migrinter explore la question des chiffres de l'immigration en publiant les travaux présentés lors de la journée d'étude du 19 octobre 2011 intitulée Les chiffres de l'immigration. Un regard critique sur les statistiques, organisée à l'Université Paris 1 par l'Institut de Démographie de l'Université Paris I (IDUP) en collaboration avec l'UMR Migrinter. Ce dossier se présente sous forme d'illustrations commentées (cartes, graphiques, etc.) extraites des diaporamas présentés lors de cette journée. À partir du cas français, mais aussi de cas européens, africains ou sud-américains, il s'agit ici de penser les catégories et les choix de la statistique publique et d'en mesurer les effets sur l'usage d'indicateurs chiffrés.

Comment mesurer les migrations ? À partir d'un premier article intitulé «Compter les migrations internationales? Quelques réflexions à propos des statistiques produites en France et en Amérique latine », Françoise Dureau interroge la notion de « résidence » au sens de rattachement au lieu qui est à la base des recensements de population et appréhende à travers elle la notion de mobilité. Ce décentrage lui permet 
de mettre en évidence la vision statique de la mobilité dans les statistiques publiques françaises ainsi que la difficulté à disposer de données statistiques de cadrage pour qui étudie les migrations en France. L'auteure montre qu'une approche moins statique prenant en compte la dimension circulatoire des mobilités et la pluralité des modes d'habiter est possible. Françoise Dureau met notamment en avant la notion de "système de résidence» qui permet de mieux comprendre l'articulation entre les lieux et les pratiques en lien avec les structures familiales, les emplois exercés et les contraintes administratives concernant la résidence.

Avec une étude sur «Les statistiques des migrations africaines: ni exode ni invasion », David Lessault et Cris Beauchemin proposent une analyse des migrations africaines loin des clichés d'invasion ou d'exode massif. Les auteurs montrent que si les migrations africaines concentrent l'essentiel de l'attention des médias et des politiques - avec notamment les accords de gestion concertés des flux migratoires - elles sont pourtant statistiquement minoritaires, en stock comme en flux, y compris en tenant compte des migrations dites «irrégulières ». La difficile question de l'estimation du nombre des retours et des allers-retours n'est pour une fois pas éludée. Les auteurs estiment qu'un réexamen du rôle central de l'Afrique dans les politiques publiques françaises et européennes en matière de migration est nécessaire.

Dans une analyse titrée «Des bénéfices et des coûts de l'immigration : les perspectives macroéconomiques d'une politique d'immigration active en France », Xavier Chojnicki étudie l'impact démographique et macroéconomique de l'immigration en lien avec le vieillissement démographique, ce qui permet de repenser les estimations des montants relatifs à l'immigration. Après avoir mesuré une contribution nette globale de l'immigration au budget des administrations publiques positive - de l'ordre de 3,9 milliards d'euros pour l'année 2005 - l'auteur utilise un modèle d'équilibre général calculable à générations imbriquées pour montrer que les effets bénéfiques de l'immigration sur les comptes de la protection sociale proviennent essentiellement de la structure par âge des flux nets, ce qui implique que cet effet positif d'une immigration sélective n'est que transitoire.

Étant unilatéralement concentrée sur l'immigration, soit sur les entrées sur le territoire, la statistique publique française ignore largement les sorties et par là, toute dynamique d'émigration. Luc Legoux et Renaud Orain constatent qu'une telle connaissance, qui ne peut être qualifiée que de partielle, induit une analyse partiale des flux migratoires. Dans l'article intitulé «Une étrange absence, la faible prise en compte des sorties dans les statistiques migratoires ", les auteurs recensent les données qui permettent de tenter une estimation des sorties. Celles-ci ne sont pas négligeables, puisque ces derniers estiment que les sorties d'étrangers représentent plus de $40 \%$ des entrées d'étrangers. Cependant, les sorties du territoire ne concernent pas que les étrangers et la prise en compte du solde migratoire négatif des Français explique une croissance du nombre des immigrés légèrement supérieure aux soldes migratoires totaux.

Enfin, ce serait dresser un bilan trop incomplet que de questionner les chiffres de l'immigration sans aborder ceux de l'enfermement des migrants. En s'interrogeant sur les centres de détention et de refoulement, Olivier Clochard s'appuie à la fois sur les données européennes et sur les données collectées au sein du réseau Migreurop pour dresser une carte des lieux d'enfermement dans l'Union européenne (420 lieux recensés), tout en soulignant la grande diversité des structures et leurs évolutions très rapides. Au-delà de cette diversité, l'auteur constate que les autorités ont recours à une multitude d'autres lieux 
qui ne figurent pas dans les listes officielles des centres de rétention administrative ce qui conduit à douter de l'exhaustivité des statistiques. L'analyse de l'enfermement sur l'île de Chypre, où l'auteur a mené une enquête, est à ce titre fort instructive.

Marianne Blidon

MCF en Démographie

Institut de Démographie de l'Université de

Paris 1 (IDUP)

UR12-INED

marianne.blidon@univ-paris1.fr

Luc Legoux

MCF en Démographie

Institut de Démographie de l'Université de

Paris 1 (IDUP)

Migrinter - UMR 7301

luc.legoux@univ-paris1.fr 